\section{Trübung des Urins}

W. G. Guder

München, Deutschland

Synonym(e) Harntrübung

Englischer Begriff urine turbidity

Definition Sichtbare Trübung des frisch gelassenen Urins im Sammelbehälter unabhängig von der Entstehung vor, während oder nach der Miktion.

Beschreibung Eine Trübung des Harns war schon in der antiken und mittelalterlichen Uroskopie (Harnschau) Gegenstand mehr oder weniger spekulativer Interpretationen. Unterschied der Uroskopiker die Trübung noch nach Wolken (nephele), Suspension (enaiorema) und Niederschlag (hypostasis), wurden im 18.-19. Jahrhundert die chemischen Eigenschaften und mit Erfindung des Mikroskops die kristal- linen Formen der $\triangleright$ Trübung unterschieden und in Beziehung zu Erkrankungen des Patienten gebracht. Von diagnostischer Bedeutung blieben Trübungen, die auf definierte Erkrankungen hindeuteten und gemeinsam mit der Farbe der Trübung, teilweise namengebend für die Substanzen oder die Erkrankung wurden (Alkaptonurie, Zystinurie oder Phosphaturie). Weitgehend ersetzt durch die spezifischen Teststreifen und chemischen Untersuchungen ist die Deutung einer Harntrübung kompliziert geworden durch artefizielle Ursachen (z. B. Kontrastmittel, Medikamente oder Nahrungszusätze). Dennoch sollte die Deutung einer Trübung des Urins jedem Arzt geläufig sein:

- Trübung des Harns im alkalischen pH-Bereich: Phosphate, Carbonate, Oxalate, Bakterien durch Verunreinigung, Schleim

- Trübung im sauren $\mathrm{pH}-$ Bereich: Urate

- Trübungen unabhängig vom pH des Urins: Schleim, Leukozyten, Epithelien, Erythrozyten (rot), Kontrastmittel, Fett (Chylurie), Medikamente ( $\triangleright$ Harnsediment) 